\title{
Tratamento de depressão em indivíduos infectados pelo HIV
}

\section{Treatment of depression in HIV-infected individuals}

\author{
André Malbergier ${ }^{\mathrm{a}, \mathrm{b}}$ e Adriana C Schöffel ${ }^{\mathrm{c}, \mathrm{d}}$

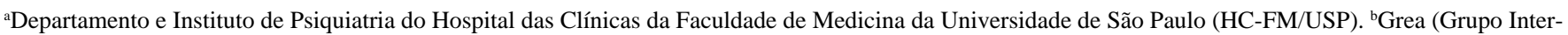 \\ disciplinar de Estudos de Álcool e Drogas). São Paulo, SP, Brasil. 'Universidade Federal do Rio Grande do Sul. 'Departamento de Psiquiatria e Medicina \\ Legal, Serviço de Psiquiatria do Hospital das Clínicas de Porto Alegre. Porto Alegre, RS, Brasil
}

Resumo A infecção pelo HIV/Aids é freqüentemente associada a transtornos psiquiátricos. Dentre eles, a depressão é o mais comum. O diagnóstico e o tratamento dos transtornos depressivos são fundamentais para melhorar a qualidade de vida desses pacientes. Esta revisão tem como objetivo sintetizar e discutir os resultados mais importantes da literatura a respeito das particularidades do tratamento dos transtornos depressivos em indivíduos infectados pelo HIV. São discutidos a epidemiologia, o quadro clínico, a influência da depressão na evolução da infecção, o tratamento farmacológico com antidepressivos, testosterona e psicoestimulantes e a interação farmacológica entre os antidepressivos e benzodiazepínicos e as drogas antivirais. Conclui-se que o tratamento antidepressivo nessa população é eficaz, seguro e não promove imunossupressão nos indivíduos afetados.

Descritores HIV. Aids. Depressão. Antidepressivos.

Abstract HIV/AIDS infection is frequently associated with psychiatric disorders, especially depression. The diagnosis and treatment of depression are essential to improve quality of life in these individuals. This review intends to summarize and discuss the most important results in the literature about the treatment of depression in HIVinfected individuals. The epidemiology, clinical presentation, contribution of depression on HIV infection, pharmacological treatment with antidepressants, testosterone, and psychostimulant drugs, and pharmacological interactions between antidepressants, benzodiazepines, and antiviral medications are discussed. The conclusion drawn from this study is that the treatment of depression in this population is effective, safe and does not promote immunosupression.

Keywords HIV. AIDS. Depression. Antidepressive agents.

\section{Introdução}

Em 1981, o Centro de Controle de Doenças ("Centers for Disease Control" - CDC) - órgão dos Estados Unidos que centraliza as normas e as ações relacionadas às doenças - notificou, pela primeira vez, a ocorrência de doenças que, retrospectivamente, foram classificadas como manifestações da Aids (síndrome da imunodeficiência adquirida). ${ }^{1}$

Essa doença caracteriza-se por um transtorno da imunidade celular, resultando em uma maior suscetibilidade a infecções oportunistas e neoplasias. ${ }^{2}$

A Organização Mundial da Saúde (OMS) estima que aproximadamente 36 milhões de pessoas estejam infectadas pelo
HIV (human immunodeficiency virus) em todo o mundo. ${ }^{3}$

No Brasil, o Ministério da Saúde contabiliza 184 mil casos de Aids notificados desde o início da epidemia. ${ }^{4}$

A Aids tem se tornado objeto de interesse por parte de psiquiatras, psicólogos e outros profissionais de saúde mental essencialmente por duas razões: o tropismo do HIV pelo sistema nervoso central (SNC) e o impacto psicológico do diagnóstico e da evolução da infecção nos indivíduos afetados. Diante disso, desenvolveram-se duas grandes áreas de interesse. A primeira situa-se nos limites da psiquiatria e da neurologia e tem como foco de interesse as consequiências clínicas da ação do HIV e de outras patologias associadas (p. ex., neurotoxoplasmose e 
neurocriptococose) no cérebro. A segunda situa-se nos limites entre a psiquiatria, a psicologia e as ciências sociais e estuda as reações psicológicas, as complicações psiquiátricas da infecção e as repercussões sociais. ${ }^{5}$

Dentre os transtornos psiquiátricos mais comumente observados em indivíduos infectados pelo HIV, a depressão é a mais prevalente, ${ }^{6}$ merecendo investigação sistemática de sintomas depressivos nessa população. Essa investigação é fundamental, já que a depressão é uma patologia com alto índice de melhora quando tratada, inclusive em indivíduos infectados pelo HIV.

Esta revisão visa discutir as particularidades do diagnóstico e do tratamento da depressão em indivíduos infectados pelo HIV.

\section{Métodos}

Para elaboração deste artigo, fez-se uma revisão sistemática da literatura pelo "Medline", utilizando os descritores "depressão" e "HIV/Aids", nos últimos sete anos (1993-2000). Foram encontrados 228 artigos, e selecionados 37. Os artigos eram incluídos se abordavam epidemiologia ou tratamento. Foram excluídos artigos que não eram de língua inglesa ou que foram publicados em revistas a que o Brasil não tem acesso. Artigos anteriores a 1993 foram obtidos por meio das referências bibliográficas dos artigos selecionados pelo "Medline".

\section{Epidemiologia e fatores de risco}

Um estudo com homossexuais masculinos infectados pelo HIV e assintomáticos observou que 35,5\% dos indivíduos avaliados apresentavam o diagnóstico de depressão ao longo da vida. ${ }^{7}$ Outros estudos também encontraram prevalências ao redor de $30 \%$ a $35 \%$ de depressão ao longo da vida entre homossexuais masculinos HIV positivos e assintomáticos. É importante destacar que esses estudos não encontraram diferenças nas taxas de prevalência entre homossexuais masculinos infectados e não infectados. A prevalência, no momento da avaliação, de depressão maior em homossexuais infectados pelo HIV é em torno de duas vezes maior do que na população geral e está dentro da variação encontrada em outras doenças crônicas (5\% a $8 \%)$. Em pacientes hospitalizados, essa prevalência é maior, variando de $30 \%$ a $40 \%{ }^{8,9}$

Em estudo realizado com uma amostra de usuários de drogas injetáveis (UDI), observou-se que $43 \%$ dos pacientes do sexo masculino infectados pelo HIV e assintomáticos apresentaram o diagnóstico de depressão ao longo da vida. ${ }^{10}$ Em dois outros estudos, ao serem admitidos para tratamento, os UDI apresentavam maior freqüência de episódios depressivos do que homossexuais masculinos. ${ }^{11,12}$

Em estudo que avaliou depressão e transtornos de personalidade, observou-se que, entre os sem diagnóstico de transtorno de personalidade, $28 \%$ dos homossexuais masculinos HIV positivos e $29 \%$ dos HIV negativos apresentaram o diagnóstico de depressão ao longo da vida. Esse número cresce para $48 \%$ entre os HIV positivos e $40 \%$ entre os HIV negativos quando apresentam pelo menos um transtorno de personalidade. ${ }^{13} \mathrm{Ou}$ seja, os homossexuais masculinos com algum transtorno de personalidade apresentaram taxas similares aos UDI.
O número de estudos em heterossexuais é pequeno quando comparado ao número de estudos em homossexuais e UDI. Em um estudo na Índia, com uma amostra de homens e mulheres heterossexuais infectados pelo HIV, $40 \%$ da amostra apresentavam um transtorno depressivo. ${ }^{14}$

Em mulheres infectadas pelo HIV, 26\% a 28\% da amostra já haviam apresentado algum episódio de depressão de início posterior ao conhecimento da sorologia positiva. ${ }^{15,16}$

Quando analisados prospectivamente, $10 \%$ a $25 \%$ dos homossexuais masculinos infectados apresentarão uma síndrome depressiva no período de 2 anos. ${ }^{17}$

A depressão maior em pacientes HIV positivos está associada a vários fatores: (1) descoberta da infecção, início dos sintomas físicos, progressão da doença e das limitações por ela impostas e as complexas questões psicossociais envolvidas; (2) invasão do sistema nervoso central pelo HIV, por infecções oportunistas ou por tumores intracranianos; (3) desencadeamento de episódio depressivo em populações vulneráveis (homossexuais e UDI). ${ }^{5,18}$

A Tabela 1 resume os fatores de risco para depressão, observados nos estudos analisados, em indivíduos infectados pelo HIV: ${ }^{19-21}$

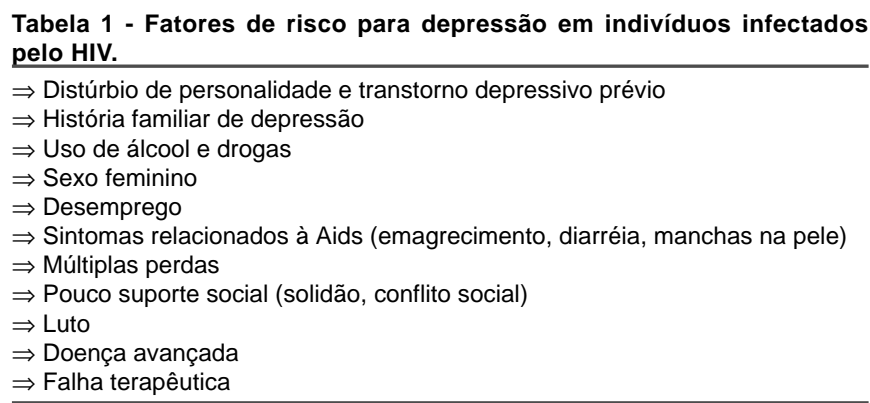

Indivíduos com Aids, com pouco suporte social, que tinham um animal de estimação referiam menos sintomas depressivos do que indivíduos que não tinham animais de estimação. Esse estudo levanta a hipótese de que animais podem também aliviar sentimentos de solidão e carência de relações pessoais nessa população. Todavia, outros estudos são necessários para investigar uma relação causal entre os dois fatores. ${ }^{22}$

\section{Quadro clínico}

O diagnóstico de depressão em pacientes infectados pode ser dificultado pelo fato de que alguns dos indicadores de depressão (anorexia, fadiga, fraqueza e perda de peso) são de pouca valia como critério diagnóstico em algumas fases da doença (especialmente na fase avançada), já que os sintomas físicos debilitantes podem mimetizar tais indicadores. Portanto, a avaliação dos sintomas de depressão deve ser criteriosa e atenta ao estágio da doença.

A Tabela 2 mostra os sintomas afetivos que devem ser valorizados na elaboração do diagnóstico de depressão e os sintomas somáticos que podem ser resultado da infecção. ${ }^{23}$

A dificuldade de distinguir sintomas depressivos de sintomas decorrentes da infecção pelo HIV é discutida em vários estudos. Perkins et $a^{24}$ concluíram que queixas de fadiga e in- 
Tabela 2 - Sintomas depressivos observados em indivíduos infectados pelo HIV.

\begin{tabular}{ll}
\hline Afetivos & Somáticos \\
\hline Humor depressivo & Alterações do apetite, perda de peso \\
Perda de interesse & Alterações de sono \\
Culpa, desvalorização & Agitação/retardo \\
Desesperança & Fadiga \\
ldeação suicida & \\
\hline
\end{tabular}

sônia em pacientes HIV positivos assintomáticos são, provavelmente, relacionadas a transtornos psíquicos, possivelmente depressão maior, sugerindo que essa patologia seja extensamente investigada na presença desses sintomas. Baker et $\mathrm{al}^{25}$ ressaltam que, apesar de haver múltiplos elementos psicossociais, etiologias orgânicas devem sempre ser consideradas em casos de pacientes em estágios avançados.

Em pesquisa visando avaliar marcadores de comprometimento orgânico e depressão, Perkins et al, ${ }^{26} \mathrm{em}$ uma amostra de homossexuais masculinos, tentaram correlacionar a depressão maior a indicadores de ação do HIV no SNC e à deficiência de vitamina $\mathrm{B}_{12}$ - em estudos anteriores, a deficiência de vitamina $\mathrm{B}_{12}$ foi associada à depressão ${ }^{27} \mathrm{e}$ à infecção pelo HIV ${ }^{28} \mathrm{Ao}$ final do estudo, concluíram: "na fase assintomática da infecção pelo HIV, depressão maior não parece ser secundária aos efeitos do HIV no SNC ou à deficiência de vitamina $\mathrm{B}_{12}{ }^{\text {". }}$.Entretanto, à medida que a infecção evolui, cresce a probabilidade de aparecimento de síndromes mentais orgânicas.

O diagnóstico diferencial entre depressão e quadros cognitivos/ demenciais pode ser de difícil realização. $\mathrm{O}$ vírus HIV produz mudanças em estruturas como gânglios da base, tálamo e lobo frontal que podem levar a transtornos da motivação e do humor. Esse processo, no início da infecção, tende a ser contido pelo próprio organismo, porém, com o avançar da doença, o próprio sistema imune, pela liberação de citocinas, pode contribuir para o aparecimento dos sintomas de uma síndrome caracterizada por: apatia, inércia, irritabilidade, alterações da memória, lentidão cognitiva, sintomas depressivos, abandono das atividades habituais, mudanças na personalidade e dificuldade em administrar conhecimentos adquiridos..$^{29}$ Esse quadro é conhecido como demência associada ao HIV-1, e os sintomas, principalmente nos estágios iniciais, podem ser semelhantes ao quadro depressivo.

$\mathrm{Na}$ infecção pelo HIV, deve-se atentar também para a indução de sintomas depressivos por medicamentos. A Tabela 3 lista os medicamentos mais comumente associados ao desencadeamento de sintomas depressivos. ${ }^{23}$

Tabela 3 - Medicações utilizadas no tratamento da infecção pelo HIV que podem induzir sintomas depressivos.

$\Rightarrow$ Esteróides: mania ou depressão

$\Rightarrow$ Interferon: depressão

$\Rightarrow$ Interleucina 2: depressão, desorientação, confusão mental

$\Rightarrow$ Zidovudine: mania, depressão

$\Rightarrow$ Efavirenz: alteração de concentração, depressão, nervosismo, pesadelos

\section{A depressão e a progressão da infecção pelo HIV}

A influência da depressão na evolução da infecção pelo HIV é objeto de vários estudos, sem todavia haver um consenso. Em acompanhamento por 8 anos de homossexuais masculi- nos infectados pelo HIV, Lyketsos et al $^{30}$ não observaram evidências de que sintomas depressivos fossem fatores de mau prognóstico. Entretanto, Burack et al ${ }^{31}$ observaram que "sintomas depressivos eram fatores preditivos de um declínio mais rápido dos níveis de linfócitos T CD4+ (um dos marcadores de progressão da doença)". Esse estudo foi realizado também em uma população de homossexuais masculinos por um período de 5 anos. Estudos mais recentes têm mostrado que a depressão pode ter um impacto na progressão da infecção pelo HIV, já que indivíduos com sintomas depressivos apresentaram, após 2 anos de seguimento, alterações imunológicas que podem apresentar implicações clínicas nessa população. ${ }^{32}$ Outro estudo em indivíduos infectados pelo HIV, mas sem Aids, acompanhados por mais de 7 anos, mostrou associação entre progressão mais rápida para Aids e maior número de eventos estressantes. Também indivíduos com mecanismos de negação de problemas e baixa satisfação com seu suporte social evoluíram mais rapidamente para Aids. Todavia, esse estudo não conseguiu demonstrar associação entre sintomas depressivos e progressão da doença. ${ }^{33}$

A literatura também é controversa a respeito da associação entre os estágios da infecção e os transtornos depressivos. Em estudo transversal, não se encontraram diferenças na prevalência dos transtornos depressivos entre os pacientes nas diversas fases da infecção. ${ }^{9}$

Em outro estudo, 112 pacientes infectados pelo HIV foram acompanhados por 4 anos em avaliações semestrais, e não se observou incremento das taxas de depressão, apesar de ter havido evolução do quadro da infecção com o aparecimento e/ou piora de sintomas físicos e também com mortes. ${ }^{34}$

No entanto, em estudo realizado com UDI, o diagnóstico de depressão e a gravidade dos sintomas depressivos mostraramse associados ao estágio sintomático da infecção pelo HIV em homens e mulheres. ${ }^{15}$ Outros dois estudos encontraram uma associação significativa entre sintomas depressivos e sintomas físicos relacionados à infecção pelo HIV. ${ }^{30,31}$

É difícil concluir definitivamente sobre a questão. Analisando os resultados dos estudos, alguns não encontraram associação entre sintomas depressivos/depressão e evolução da infecção, enquanto outros encontraram aumento da freqüência e da gravidade de transtornos depressivos à medida que a infecção evolui.

Essas diferenças e contradições, observadas nos resultados dos estudos acima descritos, revelam a dificuldade de generalização dos achados ao se estudar as alterações psicopatológicas em pacientes com comportamento de risco ou infectados pelo HIV. Além do estágio da infecção em que se encontra a casuística, a heterogeneidade dos indivíduos que apresentam comportamento de risco para a infecção pelo HIV têm sempre de ser levada em conta. O indivíduo infectado pelo HIV pode ser usuário de drogas injetáveis, homossexual ou bissexual masculino, homem ou mulher heterossexual que não utiliza preservativos em suas relações sexuais, mulher monogâmica infectada pelo seu parceiro, além de receptor de sangue ou hemoderivados. As prevalências de transtornos depressivos nessas populações são diferentes, independentemente dos indivíduos estarem infectados ou não. ${ }^{35}$ 


\section{Tratamento}

Antes de iniciar a discussão a respeito do tratamento da depressão em pacientes infectados pelo HIV, serão apresentadas, em linhas gerais, as classes de medicamentos utilizadas para o tratamento da infecção pelo HIV:

1. inibidores da transcriptase reversa análogos nucleosídeos: incorporam-se ao material genético (DNA) do vírus, resultando em DNA incompleto, incapaz de criar novos vírus. Exs.: zidovudina (AZT), didanosina (DDI), zalcitabina (DDC), estavudina (D4T), lamivudina (3TC) e abacavir;

2. inibidores da transcriptase reversa não-nucleosídeos: inibem diretamente a enzima responsável pela conversão do RNA viral para DNA, impedindo sua incorporação ao material genético da célula. Exs.: efavirenz (stocrin), nevirapina (viramune) e delavirdina (rescriptor);

3. inibidores de protease: agem no último estágio da formação do HIV, impedindo a ação da enzima protease, que é fundamental para a clivagem da cadeia protéica produzida pela célula em proteínas virais e enzimas que formarão o núcleo de cada partícula viral. Exemplos: ritonavir (norvir), nelfinavir (viracept), indinavir (crixivan), saquinavir (invirase) e amprenavir.

$\mathrm{O}$ advento dos inibidores de protease promoveu uma melhora significativa na eficácia do tratamento da infecção pelo uso do chamado "coquetel", que é constituído da combinação de mais de uma classe de medicamentos.

O tratamento da depressão apresenta algumas particularidades em pacientes HIV positivos e baseia-se em alguns princípios gerais: ${ }^{36}$

- monitorar cuidadosamente a utilização dos antidepressivos, devido ao risco de que o quadro depressivo represente infecção cerebral subclínica;

- iniciar com doses baixas e aumentá-las gradativamente para minimizar efeitos colaterais e melhorar a adesão;

- considerar a possibilidade de manutenção com doses mais baixas (os pacientes com HIV/Aids podem, principalmente nos estágios avançados, responder a doses menores que as utilizadas em indivíduos hígidos);

- atentar para as interações medicamentosas. Há possibilidade de alterações na absorção ou na excreção de medicamentos e de indução, inibição e competição pelo metabolismo por enzimas do citocromo $\mathrm{P} 450$, especialmente a família 3A3/4;

- escolher medicamentos com menos efeitos adversos ou cujos efeitos sejam benéficos para o paciente (por exemplo, constipação para pacientes com diarréia ou sedação para pacientes insones);

- atentar para os efeitos anticolinérgicos dos antidepressivos;

- ter atenção com os anti-retrovirais que o paciente venha utilizando, devido às interações dos antidepressivos e benzodiazepínicos com essas drogas.

No início da década de 90, começaram a ser publicados os primeiros estudos a respeito da eficácia e da tolerabilidade dos antidepressivos em indivíduos HIV positivos. A imipramina foi testada, em comparação ao placebo, mostrando eficácia similar aos estudos em pacientes não infectados. Os efeitos colaterais mais comuns foram relacionados à ação anticolinérgica como sedação, alterações cognitivas e tontura. ${ }^{37} \mathrm{O}$ uso de antidepressivos tricíclicos é mais tolerado na fase assintomática do que na fase avançada da infecção. Os indivíduos infectados tendem a ser mais sensíveis aos efeitos colaterais dos tricíclicos do que a população em geral. Recomendam-se, assim, cautela em sua administração e uso de drogas que tenham menos efeitos anticolinérgicos, como a nortriptilina. Os tricíclicos podem precipitar ou agravar alterações cognitivas e até quadros de delirium. $\mathrm{O}$ ressecamento de mucosa provocado por essas medicações pode facilitar o desenvolvimento de candidíase. Entretanto, os tricíclicos podem melhorar a diarréia e a insônia, sintomas comuns em pacientes infectados pelo HIV. Recomenda-se iniciar o tratamento com $25 \mathrm{mg}$ e aumentar gradativamente a dose dependendo da tolerância do paciente. Se bem tolerados, pode-se atingir dose de $300 \mathrm{mg}$ de imipramina, amitriptilina e clormipramina (150 mg para a nortriptilina) por dia. ${ }^{38}$

Os inibidores seletivos da recaptura de serotonina (p. ex., fluoxetina, sertralina, paroxetina) são mais tolerados pelos pacientes. Têm, como efeitos colaterais mais freqüentes, a redução do apetite e do peso, a insônia e a diarréia. A fluoxetina é usada na dose de $20 \mathrm{mg}$ a $80 \mathrm{mg}$ por dia. A sertralina pode ser usada em doses de $50 \mathrm{mg}$ a $200 \mathrm{mg}$ por dia, e a paroxetina, de $20 \mathrm{mg}$ a $60 \mathrm{mg}$ por dia. ${ }^{39} \mathrm{O}$ citalopram pode ser usado na dose de $10 \mathrm{mg}$ a $60 \mathrm{mg}$ por dia. A nefazodona também mostrou-se eficaz em pacientes HIV positivos e com poucos efeitos colaterais. ${ }^{40}$ A paroxetina mostrou-se tão eficaz quanto à imipramina no tratamento da depressão em pacientes HIV positivos, mas melhor tolerada, gerando menor índice de abandono de tratamento. Dos pacientes que tomaram imipramina, $48 \%$ abandonaram o tratamento contra $20 \%$ no grupo da paroxetina e $24 \%$ do grupo-placebo. É importante destacar que o abandono de tratamento é alto também no grupo que não recebeu droga ativa. Esse fato é mais freqüente nos estudos em pacientes HIV positivos do que em estudos em indivíduos não infectados. Merece destaque também que o estágio da infecção pelo HIV não influenciou a taxa de resposta ao tratamento. ${ }^{41}$

Em um estudo comparando a eficácia da fluoxetina e do placebo em indivíduos infectados pelo HIV, observou-se que 74\% dos pacientes tratados com fluoxetina responderam ao tratamento contra $47 \%$ do grupo-placebo. O estágio da doença e da imunossupressão também não foram fator determinante de resposta ao tratamento, e o tratamento com fluoxetina não foi associado a alterações na contagem de linfócitos T CD4. Os autores destacam que tanto a alta resposta ao placebo quanto a alta freqüência de abandono sugerem que outros fatores possam estar envolvidos no tratamento da depressão que não a droga em questão. Ressaltam também a dificuldade dos pacientes de tomar mais uma medicação, já que estão submetidos a regimes medicamentosos que incluem muitas drogas em várias tomadas diárias. ${ }^{42}$

A sertralina também foi estudada em pacientes HIV positivos, mostrando eficácia em $70 \%$ da amostra. Dos pacientes, $18 \%$ interromperam o uso da medicação por efeitos colaterais. Os efeitos colaterais mais comuns foram: hiperes- 
timulação $(18 \%)$, náusea (14\%), tontura (14\%) e insônia $(14 \%)$. Vale destacar que a dose média da sertralina foi 120 $\mathrm{mg}$, não se diferenciando dos estudos em pacientes não infectados pelo HIV. ${ }^{43}$

Os inibidores da MAO são evitados por apresentar riscos graves de interação medicamentosa. Lítio deve ser prescrito com cautela em pacientes com infecção por criptosporídeo, com diarréia grave ou perda importante de fluidos orgânicos.

$\mathrm{O}$ uso de metilfenidato, em um grupo seleto de deprimidos que se apresentam apáticos, fatigados, refratários a outros tratamentos ou que não toleram os efeitos colaterais dos antidepressivos, tem sido descrito como opção terapêutica. ${ }^{44} \mathrm{O}$ uso de psicoestimulantes tem como vantagem, em relação aos antidepressivos, o rápido início de ação (em geral, 2 a 3 dias). ${ }^{45}$

O uso de testosterona para tratamento de sintomas depressivos em pacientes HIV positivos vem sendo estudado mais recentemente. Essa droga foi proposta a partir de achados em pacientes infectados pelo HIV tratados com antidepressivos que, mesmo após melhora do humor depressivo, mantinham queixas residuais, como diminuição da libido, baixa energia, perda de peso e de massa muscular. Esses sintomas lembravam os apresentados por pacientes com diagnóstico de hipogonadismo que se beneficiavam de reposição com testosterona. No início da década de 90, também foi observado que a deficiência de testosterona é a alteração endócrina mais comum em pacientes infectados pelo HIV. ${ }^{46}$ Esses fatos levaram ao desenvolvimento de pesquisas com reposição de testosterona para homens infectados pelo HIV que apresentam, além do humor depressivo, diminuição da libido, fraqueza, baixa energia, perda de peso e de massa muscular. Em estudo duplo-cego controlado com placebo, indivíduos tratados com injeções de $400 \mathrm{mg}$ de cipionato de testosterona a cada duas semanas apresentaram, em relação à libido e à energia, taxas de melhora significativamente superiores ao grupo tratado com placebo. Em relação ao humor, a diferença entre os grupos ativo e placebo obteve nível de significância de $8 \%$. Houve também aumento significativo do peso no grupo da testosterona. O tratamento foi bem tolerado, embora alguns efeitos colaterais tenham sido observados: atrofia testicular $(23 \%)$, irritabilidade $(22 \%)$, acne (19\%) e dificuldade de ejaculação (10\%). ${ }^{47}$ Wagner et $\mathrm{al}^{48}$ observaram que pacientes tratados com testosterona que também realizavam exercícios físicos apresentavam, comparados aos pacientes que só recebiam testosterona, melhora significativamente superior no humor e na massa muscular. O uso de testosterona tem sido indicado tanto para pacientes com níveis séricos baixos quanto para pacientes com níveis normais. ${ }^{49}$

Em depressões graves, quando se necessita uma resposta imediata, ou em pacientes que não toleram o uso de antidepressivos, a eletroconvulsoterapia (ECT) pode ser uma opção com eficácia e tolerabilidade já comprovadas em pacientes com Aids, inclusive em casos de doença avançada. ${ }^{50}$

A Figura apresenta um fluxograma sugerido para tratamento da depressão em indivíduos infectados pelo HIV, adaptado de Barret. ${ }^{51}$

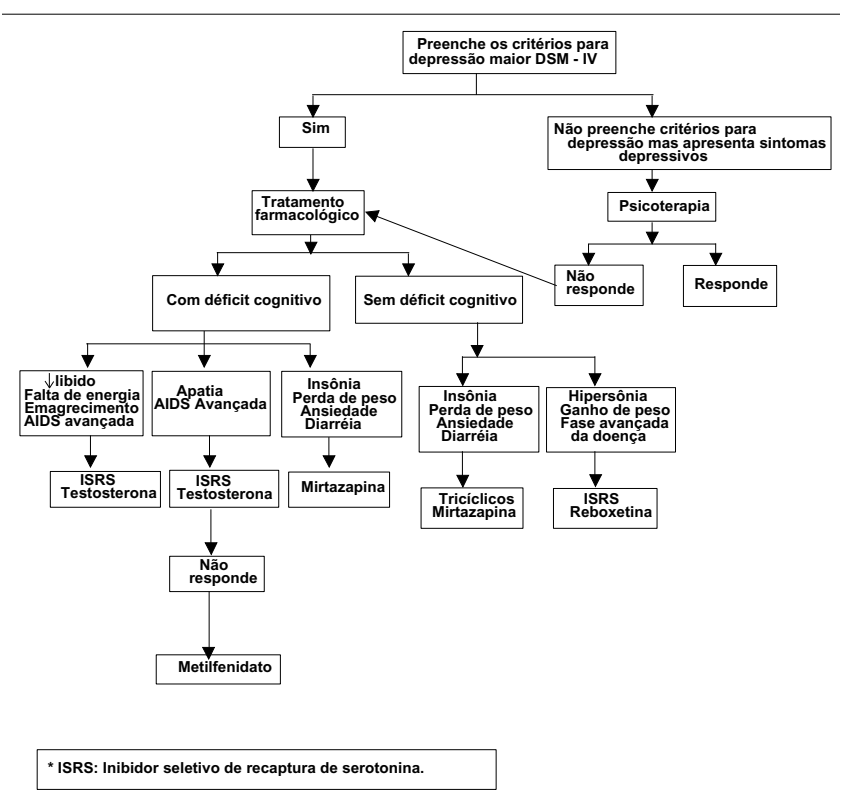

Figura - Fluxograma sugerido para tratamento da depressão em indivíduos infectados pelo HIV.

A Tabela 4 apresenta um resumo das características dos antidepressivos mais utilizados no tratamento da depressão em pacientes HIV positivos. ${ }^{52}$

Do ponto de vista clínico, a detecção e o tratamento adequado da depressão podem inclusive alterar o prognóstico do paciente. Um paciente deprimido tende a não aderir ao tratamento, a não tomar as medicações prescritas e a não acatar às orientações médicas, além de apresentar risco aumentado de suicídio. ${ }^{53}$

\section{Interação farmacológica entre antidepressivos e medicações antivirais utilizadas no tratamento da infecção pelo HIV}

Um fator importante na abordagem farmacológica da depressão em pacientes infectados é a possível interação farmacológica entre os antidepressivos e benzodiazepínicos e os medicamentos anti-HIV. Todos os inibidores de protease e a maioria dos medicamentos antidepressivos são metabolizados por enzimas do sistema citocromo P450, principalmente a família CYP3A3/4. Os inibidores de protease são também inibidores dessa família de enzimas, especialmente o ritonavir. A competição pelas vias metabólicas e a inibição da ação da enzima responsável pelo metabolismo dos medicamentos podem resultar em um aumento das concentrações séricas, resultando em um possível aumento da incidência e da intensidade dos efeitos colaterais desses medicamentos. ${ }^{40,42}$

A potência de inibição das enzimas da família CYP3A3/4 pelos inibidores de protease varia de droga para droga: ritonavir $>$ indinavir > nelfinavir > amprenavir > saquinavir.

A nefazodona deve ser usada com cuidado, pois é um inibidor potente da enzima CYP3A3/4, sendo contra-indicada em pacientes em uso de ritonavir (outro potente inibidor dessa enzima). A norfluoxetina (metabólito da fluoxetina) e a fluvoxamina são também inibidores dessa enzima, mas o uso de fluoxetina tem 
Tabela 4 - Características dos antidepressivos mais utilizados no tratamento da depressão em pacientes HIV positivos.

\begin{tabular}{|c|c|c|c|c|}
\hline Droga & $\begin{array}{l}\text { Dose } \\
\text { Inicial }\end{array}$ & $\begin{array}{l}\text { Dose } \\
\text { Terapêutica }\end{array}$ & Vantagens & $\begin{array}{l}\text { Efeitos colaterais mais comuns/ } \\
\text { Considerações }\end{array}$ \\
\hline Nortriptilina & $10-25 \mathrm{mg}$ & $50-150 \mathrm{mg}$ & $\begin{array}{l}\text { Promove o sono } \\
\text { Nível sérico é útil }\end{array}$ & Sedação e hipotensão ortostática \\
\hline Imipramina & $10-25 \mathrm{mg}$ & $100-300 \mathrm{mg}$ & $\begin{array}{l}\text { Promove sono } \\
\text { Melhora diarréia }\end{array}$ & Sedação, xerostomia, hipotensão ortostática \\
\hline Fluoxetina & $10 \mathrm{mg}$ & $20-80 \mathrm{mg}$ & Pouca sedação e efeitos colaterais & $\begin{array}{l}\text { Disfunção hepática: } \uparrow \text { níveis séricos } \\
\downarrow \text { apetite } \\
\text { Meia vida longa (metabólitos ativos) }\end{array}$ \\
\hline Sertralina & $25-50 \mathrm{mg}$ & $50-150 \mathrm{mg}$ & Pouca sedação e efeitos colaterais & Insônia, náusea, hiperestimulação e anorexia \\
\hline Amitriptilina & $10-25 \mathrm{mg}$ & $100-300 \mathrm{mg}$ & Promove o sono & $\begin{array}{l}\text { Sedação, xerostomia, obstipação, hipotensão } \\
\text { ortostática, delirium }\end{array}$ \\
\hline Paroxetina & $10 \mathrm{mg}$ & $20-60 \mathrm{mg}$ & Pouca sedação e efeitos colaterais & Insônia, náusea, anorexia, disfunção sexual \\
\hline Venlafaxina & $37,5 \mathrm{mg}$ & $75-350 \mathrm{mg}$ & Pouca sedação e efeitos colaterais & Náusea e anorexia \\
\hline Nefazodona & $100 \mathrm{mg}$ & $300-400 \mathrm{mg}$ & Pouca atuação em nível sexual & $\begin{array}{l}\text { Náusea } \\
\text { Cuidado com ritonavir }\end{array}$ \\
\hline Reboxetina & $4 \mathrm{mg}$ & 4-8 mg & $\begin{array}{l}\text { Pode ser administrado em indivíduos com } \\
\text { alterações cognitivas }\end{array}$ & $\begin{array}{l}\text { Insônia } \\
\text { Disfunção hepática: } \uparrow \text { níveis séricos }\end{array}$ \\
\hline Mirtazapina & $15 \mathrm{mg}$ & $15-45 \mathrm{mg}$ & $\begin{array}{l}\text { Promove o sono } \\
\text { Ganho de peso (pacientes emagrecidos) } \\
\text { Pouca atuação em nível sexual }\end{array}$ & $\begin{array}{l}\text { Sedação } \\
\text { Ganho de peso }\end{array}$ \\
\hline Citalopram & $10 \mathrm{mg}$ & $20-60 \mathrm{mg}$ & Pouca sedação e efeitos colaterais & Náusea, insônia \\
\hline
\end{tabular}

sido considerado seguro e eficaz nessa população. ${ }^{42}$ Citalopram, sertralina e mirtazapina parecem ser drogas seguras devido ao baixo potencial de interação farmacológica.

Entre os tricíclicos, as aminas terciárias (amitriptilina, imipramina e clomipramina) são metabolizadas (desmetiladas) pelas enzimas da família CYP3A3/4 e devem ser usadas com cautela. A nortriptilina é potencialmente mais segura.

A erva de São João e a carbamazepina são indutores do metabolismo pela família CYP 3A3/4, podendo diminuir o nível sérico dos inibidores de protease.

O uso de benzodiazepínicos, se indispensável para o tratamento da depressão, deve ser recomendado por períodos breves (2 a 3 semanas). ${ }^{53} \mathrm{~A}$ interação dos benzodiazepínicos com os inibidores de protease é outro fator limitante do uso desses medicamentos, conforme pode ser observado na Tabela 5, sendo que o lorazepam parece ser o mais seguro nessa população. ${ }^{54}$

\section{O tratamento psicoterápico da depressão em indivíduos infectados pelo HIV}

Existe um número muito menor de estudos a respeito do tratamento psicoterápico da depressão em pacientes infectados pelo HIV. Vários estudos discutem técnicas e abordagens psi- coterápicas de indivíduos infectados pelo HIV de forma genérica, citando dinâmicas, dificuldades e características comuns a essa população, sem especificar a eficácia e a indicação da psicoterapia para o tratamento da depressão em si.

Um dos estudos avaliados comparou a psicoterapia interpessoal (mais diretiva) e a de suporte. Ambas foram capazes de reduzir significativamente os escores de escalas de sintomas depressivos, embora os resultados obtidos pela psicoterapia interpessoal tenham sido superiores. Existe uma tendência da literatura de considerar que estratégias específicas de como lidar com a doença são mais eficazes na redução de sintomas ansiosos e depressivos do que outras modalidades. ${ }^{55,56}$ As intervenções específicas caracterizam-se por técnicas de solução de problemas, educação em saúde, treinamento de técnicas de relaxamento e suporte emocional.

Outro estudo comparou a eficácia de quatro modalidades terapêuticas diferentes para o tratamento de sintomas depressivos em pacientes infectados pelo HIV: psicoterapia interpessoal, cognitiva-comportamental, suportiva e suportiva com o uso de imipramina por um período de 16 semanas. Pacientes tratados com psicoterapia interpessoal e com psicoterapia suportiva com imipramina tiveram resultados significativamente

Tabela 5 - Interação farmacológica entre benzodiazepínicos e os anti-retrovirais.

\begin{tabular}{|c|c|c|c|c|c|c|c|c|c|c|c|c|}
\hline & AZT & DDI & DDC & D4T & 3TC & Indinavir & Nelfinavir & Ritonavir & Saquinavir & Delavirdina & Efavirenz & Nevirapina \\
\hline Alprazolam & $\bullet$ & • & • & $\bullet$ & $\bullet$ & $\bullet$ & + & $\bullet$ & + & $\bullet$ & + & + \\
\hline Clonazepam & $\bullet$ & - & $\bullet$ & $\bullet$ & $\bullet$ & + & + & 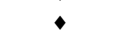 & + & + & + & + \\
\hline Diazepam & $\bullet$ & $\bullet$ & • & $\bullet$ & $\bullet$ & + & + & 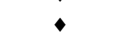 & + & + & + & $\square$ \\
\hline Estazolam & $\bullet$ & $\bullet$ & $\bullet$ & $\bullet$ & $\bullet$ & + & + & $\bullet$ & + & + & + & + \\
\hline Flurazepam & - & $\bullet$ & $\bullet$ & $\bullet$ & $\bullet$ & + & + & $\bullet$ & + & + & + & 口 \\
\hline Midazolam & - & - & - & $\bullet$ & $\bullet$ & $\bullet$ & $\bullet$ & - & - & $\bullet$ & $\bullet$ & + \\
\hline Trizolam & $\bullet$ & $\bullet$ & $\bullet$ & $\bullet$ & $\bullet$ & $\bullet$ & $\bullet$ & $\bullet$ & $\bullet$ & $\bullet$ & $\bullet$ & + \\
\hline Zolpidem & $\bullet$ & $\bullet$ & $\bullet$ & $\bullet$ & $\bullet$ & + & + & $\bullet$ & + & + & + & + \\
\hline
\end{tabular}

- Não devem ser co-administrados

+ Potencial interação que requer estreito monitoramento, alteração da dose ou intervalo de administração

- Não há interação clínica significativa

- Interações provavelmente inexistentes ou não descritas até o momento

$A Z T=$ Zidovudina

$D D I=$ Didanosina

$D D C=$ Zalcitabina

$D 4 T=$ Estavudina

$3 T C=$ Lamivudina 
superiores aos obtidos por outras modalidades terapêuticas. ${ }^{57}$

Alguns autores consideram a terapia de grupo como a modalidade mais indicada em pacientes infectados pelo HIV. Esse conceito surgiu baseado nas semelhanças entre as reações psicológicas de pacientes infectados pelo HIV. O grupo propicia tentativas saudáveis de lidar com a estigmatização e a desesperança por meio do contato com outros que estão passando por situações semelhantes e que podem ter desenvolvido estratégias mais positivas e saudáveis que podem servir com exemplo.

Os objetivos do tratamento psicoterápico são: ${ }^{58}$

- ajudar o paciente a manter o controle sobre sua vida e assisti-lo no processo de desenvolver habilidades para lidar de forma positiva com os desafios e as complicações de sua doença;

- auxiliar o paciente a lidar com os sentimentos de raiva, negação, pânico e desespero observados em alguns momentos da infecção;

- trabalhar os sentimentos de respeito próprio, culpa, autoacusação e vergonha;

- auxiliar os pacientes a estabelecer um canal de comunicação com familiares, parceiros, amigos, emprego a respeito de sua doença e do medo de rejeição, abandono e isolamento;

- ajudar os pacientes a lidar com as prováveis crises (agudizações) de sua doença;

- propiciar um espaço para o paciente expor suas questões a respeito do desfecho de sua doença e da morte.
Grupos de mulheres têm sido considerados mais eficazes para abordagem dessa subpopulação devido a particularidades quanto ao gênero e aos cuidados com os filhos. ${ }^{59}$

No Brasil, Schöffel et al ${ }^{60}$ discutem os objetivos e as técnicas de tratamento psicoterápico em pacientes infectados pelo HIV.

\section{Conclusões}

Diante das evidências discutidas neste artigo, conclui-se que:

1. a prevalência dos transtornos depressivos em homossexuais masculinos infectados pelo HIV não difere da observada em homossexuais masculinos não infectados;

2. usuários de drogas injetáveis infectados pelo HIV apresentam prevalência de transtornos depressivos maior do que homossexuais masculinos também infectados;

3. sintomas decorrentes da infecção pelo HIV podem mimetizar sintomas depressivos nos estágios avançados da infecção;

4. estudos a respeito da eficácia do tratamento dos transtornos depressivos em pacientes infectados pelo HIV demonstram que os resultados obtidos não diferem dos observados em amostras de indivíduos não infectados pelo HIV;

5. atentando-se para as interações farmacológicas, o tratamento com os antidepressivos estudados tem se mostrado seguro, não promovendo imunossupressão;

6. metilfenidato e testosterona podem ser úteis no tratamento de pacientes que não respondem à terapêutica tradicional.

\section{Referências}

1. Fenton TW. AIDS-related psychiatric disorder. Br J Psychiatry 1987;151:579-88.

2. Bowen DL, Lane HC, Fanci AS. Immunopathogenesis of the acquired immunodeficiency syndrome. Ann Int Med 1985;103:704-9.

3. World Health Organization. Epidemiologic Report, No 3, Geneve, 2000.

4. Ministério da Saúde. Boletim Epidemiológico, Ano XIII, No 1, Semana 48/99 a 22/00, 2000.

5. Maj M. Psychiatric aspects of HIV-1 infecton and AIDS. Psychol Med 1990;20:547-63.

6. Seth R, Granville-Grossman K, Goldmeier D, Lynch S. Psychiatric illnesses in patients with HIV infection and AIDS referred to the liaison psychiatrist. Br J Psychiatry 1991;159:347-50.

7. Atkinson JH, Grant I, Kennedy CJ, Richman DD, Spector AS, McCutchan A. Prevalence of psychiatric disorders among men infected with human immunodeficiency virus. A controlled study. Arch Gen Psychiatry 1988;45:859-64.

8. Williams JB, Rabkin JG, Remien RH, Gorman JM, Ehrhardt AA. Multidisciplinary baseline assessment of homosexual men with and without human immunodeficiency virus infection. II. Standardized clinical assessment of current and lifetime psychopathology. Arch Gen Psychiatry 1991;48:124-30.

9. Rabkin JG, Ferrando SJ, Jacobsberg LB, Fishman B. Prevalence of axis I disorders in an AIDS cohort: a cross-sectional, controlled study. Compr Psychiatry 1997;38:146-54.

10. Malbergier A, Andrade AG. Depressive disorders and suicide attempts in injecting drug users with and without HIV infection. AIDS Care 2000;13:141-50.

11. Lyketsos CG, Fishman M, Treisman G. Psychiatric issues and emergencies in HIV infection. Med Clin N Am 1995;13:163-77.

12. Malbergier A, Menko AC. Depressão em pacientes infectados pelo HIV: comparação entre usuários de drogas injetáveis e homossexuais masculinos. In: Simpósio Internacional "Depressão no Ciclo da Vida". Livro de Referências e Posters. São Paulo; 1996. p. 441.

13. Johnson JG, Williams DSW, Rabkin JG, Goetz RR, Remien RH. Axis I psychiatric symptoms associated with HIV infection and personality disorder. Am J Psychiatry 1995;152:551-4.

14. Chandra PS, Ravi V, Desai A, Subbakrishna DK. Anxiety and depression among HIV-infected hetrosexuals - a report from India. J Psychosom Res 1998;45:401-9.

15. Lipsitz JD, Williams JBW, Rabkin JG, Remien RH, Bradbury M, Sadr W EL, et al. Psychopathology in male and female intravenous drug users with and without HIV infection. Am J Psychiatry 1994;151:1662-8.

16. Lovisi GM, Morgado AF. Suporte social e transtornos psiquiátricos em mulheres infectadas pelo HIV. J Bras Psiquiatria 1996;45:593-6.

17. Atkinson JH, Grant I. Natural history of neuropsychiatric manifestations of HIV disease. Psych Clin N Am 1994;17:17-33.

18. Maj M. Depressive syndromes and symptoms in subjects with human immunodeficiency virus (HIV) infection. Br J Psychiatry 1996;168(Suppl 30):117-22.

19. Leserman J, DiSantostefano R, Perkins DO, Murphy C, Golden RN, Evans DL. Longitudinal study of social support and social conflict as predictors of depression and dysphoria among HIV-positive and HIV-negative gay men. Depression 1995;2:189-99.

20. Nott KH, Vedhara K, Power MJ. The role of social support in HIV infection. Psychol Med 1995;25:971-83.

21. Summers J, Zissok S, Atkinson JH, Sciolla A, Whitehall W, Brown S, et al. Psychiatric morbidity associated with acquired immune deficiency syndrome - related grief resolution. J Nerv Ment Dis 1995;183:384-9. 
22. Siegel JM, Angulo FJ, Detels R, Wesch J, Mullen A. AIDS diagnosis and depression in the Multicenter AIDS Collaborative Study: the ameliorating impact of pet ownership. AIDS Care 1999;11:157-70.

23. Ferrando S. HIV-related mood Disorders. In: The treatment of psychiatrically ill HIV patients. Chicago: American Psychiatric Association; 2000. p. 1-22.

24. Perkins DO, Leserman J, Stern RA, Baum SF, Liao D, Golden RN, et al. Somatic symptoms and HIV infection: relationship to depressive symptoms and indicators of HIV disease. Am J Psychiatry 1995;152:1776-81.

25. Baker J, Ruiz-Rodrigues R, Whitfeld M, Heon V, Berger TG. Bacillary angiomatosis: a treatable cause of acute psychiatric symptoms in human immunodeficiency virus infection. J Clin Psychiatry 1995;56:161-6.

26. Perkins DO, Stern RA, Golden RN, Murphy C, Naftolowitz D, Evans DL. Mood disorders in HIV infection: prevalence and risk factors in a nonepicenter of the AIDS epidemic. Am J Psychiatry 1994;151:233-6.

27. Evans DL, Edelsohn GA, Golden RN. Organic psychosis without anemia or spinal chord symptoms in vitamin B12 deficiency. Am J Psychiatry 1983;140:218-21.

28. Beach RS, Morgan R, Wilkie F, Mantero-Atienza E, Blaney N, Shor-Posner $\mathrm{G}$, et al. Specific nutrient abnormalities in asymptomatic HIV-1 infection. AIDS 1992;6:701-8.

29. Adams MA, Ferraro FR. Acquired immunodeficiency syndrome dementia complex. J Clin Psychology 1997;53:767-78.

30. Lyketsos CG, Hoover DR, Guccione M, Senterfitt W, Dew MA, Wesch J, et al. Depressive symptoms as predictors of medical outcomes in HIV infection. JAMA 1993;270:2563-7.

31. Burack JH, Barret DC, Stall RD, Chesney MA, Ekstrand ML, Coates TJ. Depressive symptoms and CD4 lymphocyte decline among HIV-infected men. JAMA 1993;270:2568-73.

32. Leserman J, Petito, JM, Perkins DO, Folds JD, Golden RN, Evans DL. Severe stress, depressive symptoms, and changes in lymphocytes subsets in human immunodeficiency virus infected men. A 2-year follow-up study. Arch Gen Psychiatry 1997;54:279-85.

33. Leserman J, Petito JM, Golden, RN, Gaynes BN, Gu H, Perkins DO, et al. Impact of stressful life events depression, social support, coping, and cortisol on progression to AIDS. Am J Psychiatry 2000;157:1221-8.

34. Rabkin JG, Goetz RR, Remien RH, Williams JBW, Todak G, Gorman JM. Stability of mood despite illness progression in a group of homosexual men. Am J Psychiatry 1997;154:231-8.

35. Gala C, Pergami A, Catalán J, Durbano F, Musiccio M, Baldeweg T, et al. The psychosocial impact of HIV infection in gay men, drug users and heterosexuals. Br J Psychiatry 1993;163:651-9.

36. American Psychiatric Association/AIDS Program Office. HIV-Related Neuropsychiatric Complications and Treatment. Washington (DC): APA; 1998.

37. Rabkin JG, Rabkin R, Harrison W, Wagner G. Effect of imipramine on mood and enumerative measures of immune status in depressed patients with HIV illness. Am J Psychiatry 1994;151:516-23.

38. Rabkin JG, Harrison WM. Effect of imipramine on depression and immune status in a sample of men with HIV infection. Am J Psychiatry 1990;147:495-7.

39. Ferrando SJ, Goldman JD, Charness WE. Selective serotonin reuptake inhibitor treatment of depression in symptomatic HIV infection and AIDS. Improvements in affective and somatic symptoms. Gen Hosp Psychiatry 1997;19:89-97.

40. Elliot AJ, Russo J, Bergam K, Claypoole K, Uldall KK, Roy-Byrne PP. Antidepressant efficacy in HIV-seropositive outpatients with major depressive disorder: an open trial of nefazodone. J Clin Psychiatry 1999;60:226-31.

41. Elliott AJ, Uldall KK, Bergam K, Russo J, Claypoole K, Roy-Byrne PP. Randomized, placebo-contolled trial of paroxetine versus imipramine in depressed HIV-positive outpatients. Am J Psychiatry 1998;155:367-72.

42. Rabkin JG, Wagner GJ, Rabkin R. Fluoxetine treatment for depression in patients with HIV and AIDS: a randomized, placebo-controlled trial. Am J Psychiatry 1999;156:101-7.

43. Rabkin JG, Wagner G, Rabkin R. Effects of sertraline on mood and immune status in patients with major depression and HIV illness: an open trial. J Clin Psychiatry 1994;55:433-9.
44. Fernandez F, Levy JK, Ruiz P. The use of methylfenidate in HIV patients: a clinical perspective. In: Grant I, Martin A, editors. Neuropsychology of HIV infection. New York: Oxford University Press; 1994. p. 295-309.

45. Wagner GJ, Rabkin J, Rabkin R. A comparative analysis of standard and alternative antidepressants in the treatment of human immunodeficiency virus patients. Compr Psychiatry 1996;37:402-8.

46. Raffi F, Brisseau J, Planchon B, Remi J, Barrier J, Grolleau J. Endocrine function in $98 \mathrm{HIV}$-infected patients: a prospective study. AIDS 1991;5:729-33.

47. Rabkin JG, Wagner GJ, Rabkin R. A double-blind, placebo-controlled trial of testosterone therapy for HIV-positive men with hypogonadal symptoms. Arch Gen Psychiatry 2000;57:141-7.

48. Wagner G, Rabkin J, Rabkin R. Exercise as a mediator of psychological and nutritional effects of testosterone therapy in HIV+ men. Med Sci Sports Exerc 1998;30:811-7.

49. Grinspoon S, Corcoran C, Stanley T, Baaj A, Basgoz N, Klibanski A. Effects of hypogonadism and testosterone administration on depression indices in HIV-infected men. J Clin Endocrinol Metab 2000;85:60-5.

50. Schaerf FW, Miller RR, Lipsey JR. ECT for major depression in four patients infected with human immunodeficiency virus. Am J Psychiatry 1989;146:782-4

51. Barret JG. Medical management of HIV infection. Baltimore: Port City Press, 1998.

52. Schoffel AC, Abreu PSB, Sprinz E, Tostes M. Transtornos depressivos associados à infecção pelo HIV. In: Fraguas Jr-R, Figueiró JAB, eds. Depressões secundárias: terapêuticas diferenciadas. São Paulo: Atheneu; 2001. p. 259-68.

53. Fernandez F, Levy JK. Psychiatric diagnosis and pharmacoterapy of patients with HIV infection. In: Tasman A, Goldfinger SM, Kaufmann CA, editors. Review of Psychiatry. Washington (DC): American Psychiatric Press Inc.; 1990. p. 614-30.

54. Ministério da Saúde. Consenso sobre terrapia anti-retrovira para adultos e adolescentes infectados pelo HIV. Brasília, 1998.

55. Markowitz JC, Klerman GL, Clougherty KF, Spielman LA, Jacobsberg LB, Fishman B, et al. Individual psychotherapies for depressed HIVpositive patientes. Am J Psychiatry 1995;152:1504-9.

56. Kelly JA, Murphy DA, Bahr GR, Kalichman SC, Morgan MG, Stevenson LY, et al. Outcome of cognitive-behavioral and support group therapies for depressed, HIV-infected persons. Am J Psychiatry 1993;150:1679-86.

57. Markowitz JC, Kocsis JH, Fishman B, Spielman LA, Jacobsberg LB, Frances AJ, et al. Treatment of depressive symptoms in human immunodeficiency virus-positive patients. Arch Gen Psychiatry 1998;55:452-7.

58. Zegans LS, Gerhard AL, Coates TJ. Psychoterapies for the person with HIV disease. In: Zegans LS, Coates TJ, eds. Psychiatric Manisfetations of HIV disease. The Psychiatric Clinics of North America. Philadelphia: W B Saunders Company; 1994. p. 149-162.

59. Chung JY, Magraw MM. A group approach to psychosocial issues faced by HIV-positive women. Hosp Community Psychiatry 1992;43:891.

60. Schöffel AC, Abreu PBS, Tatsch MF. Psicoterapia para pacientes infectados pelo HIV. Rev Psiquiatr RS 1997;19:28-33.

Correspondência

André Malbergier

Grea - Instituto de Psiquiatria do Hospital das Clínicas da FM/USP

Rua Dr. Ovídio Pires de Campos $s / n^{\circ}$

05403-010 São Paulo, SP

Tel: (0xx11) 3081-8060

Fax: (0xx11) 3064-4973

E-mail: malbergiera@originet.com.br e grea@edu.usp.br 